\title{
Appendix A: Miscellaneous tables
}

Table A. Word list used for native Southern French production data and stimulus elicitation.

\begin{tabular}{|c|c|c|c|c|c|c|c|c|}
\hline \multirow{2}{*}{$\begin{array}{c}\text { Onset } \\
\mathrm{C}\end{array}$} & \multicolumn{8}{|c|}{ Target V } \\
\hline & /a/ & $/ \tilde{\mathrm{a}} /$ & $|\varepsilon|$ & $\mid \tilde{\varepsilon} /$ & /o/ & $/ \tilde{\partial} /$ & /i/ & $/ \mathrm{u} /$ \\
\hline$/ \mathrm{p} /$ & $\begin{array}{c}\text { pas } \\
\text { "step" }\end{array}$ & $\begin{array}{l}\text { paon } \\
\text { "peacock" }\end{array}$ & $\begin{array}{c}\text { paix } \\
\text { "peace" }\end{array}$ & $\begin{array}{c}\text { pain } \\
\text { "bread" }\end{array}$ & $\begin{array}{c}\text { pot } \\
\text { "pot/jar" }\end{array}$ & $\begin{array}{c}\text { pont } \\
\text { "bridge" }\end{array}$ & $\begin{array}{c}\text { pie } \\
\text { "magpie" }\end{array}$ & $\begin{array}{c}\text { pou } \\
\text { "louse" }\end{array}$ \\
\hline$/ \mathrm{b} /$ & $\begin{array}{l}\text { bas } \\
\text { "low" }\end{array}$ & $\begin{array}{c}\text { banc } \\
\text { "bench" }\end{array}$ & $\begin{array}{l}\text { baie } \\
\text { "bay" }\end{array}$ & $\begin{array}{l}\text { bain } \\
\text { "bath" }\end{array}$ & $\begin{array}{l}\text { beau } \\
\text { "beautiful }\end{array}$ & $\begin{array}{c}\text { bon } \\
\text { "good" }\end{array}$ & $\begin{array}{c}b i \\
\text { "bi/dual" }\end{array}$ & $\begin{array}{c}\text { bout } \\
\text { "tip/end" }\end{array}$ \\
\hline
\end{tabular}

Table B. Maximum formant values $(\mathrm{Hz})$ for automatic estimation of the first two formants in Praat.

\begin{tabular}{c|cccc|cccccccccc}
\hline \multirow{2}{*}{ Vowel pair } & \multicolumn{9}{|c|}{ Native speakers } & \multicolumn{10}{|c}{ Naïve imitators } \\
& SF01 & SF02 & SF03 & SF04 & AE01 & AE02 & AE03 & AE04 & AE05 & AE06 & AE07 & AE08 & AE09 \\
\hline$/ \tilde{\varepsilon} /-/ \varepsilon /$ & 2000 & 2000 & 2000 & 1600 & 1600 & 1400 & 1200 & 1450 & 1400 & 1350 & 1400 & 1550 & 1300 \\
$/ \tilde{\mathrm{a}} /-/ \mathrm{a} /$ & 2000 & 1600 & 1800 & 1800 & 1600 & 1350 & 1400 & 1500 & 1450 & 1300 & 1500 & 1600 & 1800 \\
$/ \tilde{\mathrm{s} /-/ \mathrm{o} /}$ & 1500 & 1200 & 1500 & 1600 & 1600 & 1500 & 1300 & 1500 & 1700 & 1700 & 1350 & 1250 & 1650 \\
\hline
\end{tabular}


Table C. Results for linear mixed-effects models created to test the effect of nasality on F1', nasalance, tongue height, and contact quotient. For each vowel pair model, the oral vowel is used as the base model and the estimates are for the nasal vowel in comparison to its oral counterpart. Random intercepts for imitator, speaker, token order, and onset consonant are included in each model. Statistically significant effects are highlighted in gray for Bonferroni adjusted $\alpha=0.004$.

\begin{tabular}{|c|c|c|c|c|c|c|c|}
\hline DV & Comparison & Inter. & $\beta$ & $S E$ & $d f$ & $t$ & $p$ \\
\hline \multirow{3}{*}{$\mathrm{F} 1^{\prime}$} & / $\tilde{\mathrm{a}} /$ vs. /a/ & 1.429 & -0.860 & 0.036 & 257.9 & -23.98 & $<0.001$ \\
\hline & $/ \tilde{\varepsilon} /$ vs. $/ \varepsilon /$ & -0.176 & 0.842 & 0.037 & 304.9 & 23.05 & $<0.001$ \\
\hline & 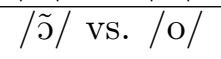 & -0.275 & 0.006 & 0.029 & 275.8 & 0.20 & $=0.840$ \\
\hline \multirow{3}{*}{ Nasalance } & /ã / vs. /a/ & -1.075 & 1.914 & 0.029 & 206.0 & 65.76 & $<0.001$ \\
\hline & $/ \tilde{\varepsilon} /$ vs. $/ \varepsilon /$ & -0.561 & 1.440 & 0.028 & 220.2 & 51.41 & $<0.001$ \\
\hline & 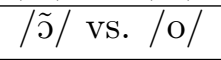 & -0.781 & 1.971 & 0.036 & 220.9 & 54.51 & $<0.001$ \\
\hline \multirow{3}{*}{ Height } & /ã / vs. /a/ & -1.072 & 0.031 & 0.023 & 685.1 & 1.34 & $=0.181$ \\
\hline & $/ \tilde{\varepsilon} /$ vs. $/ \varepsilon /$ & 0.831 & -1.133 & 0.028 & 468.9 & -40.2 & $<0.001$ \\
\hline & 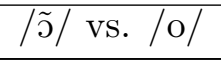 & -0.096 & -0.403 & 0.026 & 602.0 & -15.57 & $<0.001$ \\
\hline \multirow{3}{*}{ CQ } & /ã / vs. /a/ & 0.332 & -0.334 & 0.046 & 304.2 & -7.21 & $<0.001$ \\
\hline & $/ \tilde{\varepsilon} /$ vs. $/ \varepsilon /$ & 0.323 & -0.217 & 0.053 & 374.2 & -4.07 & $<0.001$ \\
\hline & 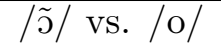 & 0.040 & -0.299 & 0.055 & 341.4 & -5.45 & $<0.001$ \\
\hline
\end{tabular}

Table D. Results for linear mixed-effects models created to test the effect of group on nasal-oral difference in $\mathrm{F}^{\prime}$, nasalance, tongue height, and contact quotient. For each vowel pair model, the SF native speakers are used as the base model and the estimates are for the AE naïve imitators in comparison to the SF speakers.

Random intercepts for imitator, speaker, token order, and onset consonant are included in each model. Statistically significant effects are highlighted in gray for Bonferroni adjusted $\alpha=0.004$.

\begin{tabular}{|c|c|c|c|c|c|c|c|}
\hline DV & Difference & Inter. & $\beta$ & $S E$ & $d f$ & $t$ & $p$ \\
\hline \multirow{3}{*}{$\mathrm{F} 1^{\prime}$} & $/ \tilde{\mathrm{a}} /-/ \mathrm{a} /$ & -0.819 & -0.034 & 0.051 & 1194.7 & -0.66 & $=0.508$ \\
\hline & $\mid \tilde{\varepsilon} /-/ \varepsilon /$ & 0.914 & -0.140 & 0.052 & 1270.0 & -2.72 & $=0.007$ \\
\hline & $\mid \tilde{\mathrm{o}} /-/ \mathrm{o} /$ & 0.012 & 0.003 & 0.038 & 1178.2 & 0.07 & $=0.944$ \\
\hline \multirow{3}{*}{ Nasalance } & $/ \tilde{\mathrm{a}} /-/ \mathrm{a} /$ & 1.984 & -0.149 & 0.046 & 1189.4 & -3.25 & $<0.004$ \\
\hline & $\mid \tilde{\varepsilon} /-/ \varepsilon /$ & 1.672 & -0.492 & 0.043 & 1209.0 & -11.47 & $<0.001$ \\
\hline & $\mid \tilde{\mathrm{o}} /-/ \mathrm{o} /$ & 2.331 & -0.752 & 0.051 & 1184.0 & -14.74 & $<0.001$ \\
\hline \multirow{3}{*}{ Height } & $/ \tilde{\mathrm{a}} /-/ \mathrm{a} /$ & -0.044 & 0.162 & 0.024 & 1191.7 & 6.78 & $<0.001$ \\
\hline & $\mid \tilde{\varepsilon} /-/ \varepsilon /$ & -1.072 & -0.186 & 0.029 & 1211.9 & -6.50 & $<0.001$ \\
\hline & $\mid \tilde{\mathrm{o}} /-/ \mathrm{o} /$ & -0.506 & 0.110 & 0.025 & 1183.2 & 4.33 & $<0.001$ \\
\hline \multirow{3}{*}{ CQ } & $/ \tilde{\mathrm{a}} /-/ \mathrm{a} /$ & -0.336 & 0.036 & 0.060 & 1188.3 & 0.61 & $=0.542$ \\
\hline & $|\tilde{\varepsilon} /-| \varepsilon /$ & -0.152 & -0.137 & 0.063 & 1216.4 & -2.19 & $=0.029$ \\
\hline & $\mid \tilde{\mathrm{o}} /-/ \mathrm{o} /$ & -0.262 & 0.045 & 0.064 & 1183.2 & 0.71 & $=0.478$ \\
\hline
\end{tabular}


Table E. Results for linear mixed-effects models created to test the effect of nasalance, tongue height, and contact quotient on $\mathrm{F}^{\prime}$, as well as the interaction of group with these articulatory variables. For each vowel pair model, the SF native speakers are used as the base model and the estimates are for the AE naïve imitators in comparison to the SF speakers. Random intercepts for imitator, speaker, token order, and onset consonant are included in each model. Statistically significant effects are highlighted in gray for Bonferroni adjusted $\alpha=0.006$.

\begin{tabular}{|c|c|c|c|c|c|c|}
\hline Difference & Factor & $\beta$ & $S E$ & $d f$ & $t$ & $p$ \\
\hline \multirow{9}{*}{$/ \tilde{\mathrm{a}} /-/ \mathrm{a} /$} & Nasalance & -0.662 & 0.046 & 1250.0 & -14.49 & $<0.001$ \\
\hline & Group & -0.489 & 0.118 & 1255.1 & -4.14 & $<0.001$ \\
\hline & Nasal. : Gr. & 0.194 & 0.057 & 1252.9 & 3.43 & $<0.001$ \\
\hline & Height & 0.089 & 0.102 & 747.9 & 0.87 & $=0.384$ \\
\hline & Group & -0.006 & 0.051 & 1203.6 & -0.13 & $=0.899$ \\
\hline & Height : Gr. & -0.327 & 0.117 & 992.2 & -2.81 & $<0.006$ \\
\hline & $\mathrm{CQ}$ & 0.278 & 0.036 & 1073.4 & 7.61 & $<0.001$ \\
\hline & Group & -0.090 & 0.052 & 1216.3 & -1.73 & $=0.084$ \\
\hline & CQ : Gr. & -0.157 & 0.048 & 1150.6 & -3.28 & $<0.006$ \\
\hline \multirow{9}{*}{$|\tilde{\varepsilon}|-\mid \varepsilon /$} & Nasalance & -0.642 & 0.054 & 1271.9 & -11.98 & $<0.001$ \\
\hline & Group & -0.743 & 0.110 & 1271.4 & -6.73 & $<0.001$ \\
\hline & Nasal. : Gr. & 0.243 & 0.065 & 1271.6 & 3.73 & $<0.001$ \\
\hline & Height & 0.329 & 0.081 & 1264.5 & 4.04 & $<0.001$ \\
\hline & Group & -0.478 & 0.115 & 1272.0 & -4.14 & $<0.001$ \\
\hline & Height : Gr. & -0.316 & 0.091 & 1272.8 & -3.46 & $<0.001$ \\
\hline & $\mathrm{CQ}$ & 0.134 & 0.034 & 1271.1 & 3.93 & $<0.001$ \\
\hline & Group & -0.126 & 0.052 & 1269.1 & -2.45 & $=0.015$ \\
\hline & CQ : Gr. & -0.018 & 0.044 & 1271.0 & -0.41 & $=0.682$ \\
\hline \multirow{9}{*}{$\mid \tilde{\mathrm{o}} /-/ \mathrm{o} /$} & Nasalance & -0.435 & 0.030 & 1237.6 & -14.31 & $<0.001$ \\
\hline & Group & -0.635 & 0.088 & 1235.0 & -7.18 & $<0.001$ \\
\hline & Nasal. : Gr. & 0.197 & 0.040 & 1239.8 & 4.97 & $<0.001$ \\
\hline & Height & 0.092 & 0.076 & 1072.4 & 1.22 & $=0.223$ \\
\hline & Group & -0.110 & 0.056 & 1244.4 & -1.97 & $=0.049$ \\
\hline & Height : Gr. & -0.263 & 0.086 & 1210.2 & -3.06 & $<0.006$ \\
\hline & $\mathrm{CQ}$ & 0.130 & 0.023 & 1161.6 & 5.69 & $<0.001$ \\
\hline & Group & 0.002 & 0.038 & 1183.8 & 0.06 & $=0.953$ \\
\hline & CQ : Gr. & 0.026 & 0.033 & 1232.3 & 0.79 & $=0.432$ \\
\hline
\end{tabular}

